\title{
MULTI-TEMPORAL SAR INTERFEROMETRY FOR LANDSLIDE MONITORING
}

\author{
R. Dwivedi ${ }^{a^{*}}$, A. B. Narayan ${ }^{\text {b }}$, A. Tiwari ${ }^{\text {b }}$, O. Dikshit ${ }^{\text {b }}$, A. K. Singh ${ }^{\text {a }}$ \\ ${ }^{a}$ Geographic Information System (GIS) Cell, MNNIT Allahabad, India - (ramjid, aksingh)@mnnit.ac.in \\ ${ }^{\mathrm{b}}$ Department of Civil Engineering, IIT Kanpur, India - (avadhbn, ashut, onkar)@iitk.ac.in
}

\section{Commission VIII, WG VIII/1}

KEY WORDS: SAR Interferometry, Landslide monitoring

\begin{abstract}
:
In the past few years, SAR Interferometry specially InSAR and D-InSAR were extensively used for deformation monitoring related applications. Due to temporal and spatial decorrelation in dense vegetated areas, effectiveness of InSAR and D-InSAR observations were always under scrutiny. Multi-temporal InSAR methods are developed in recent times to retrieve the deformation signal from pixels with different scattering characteristics. Presently, two classes of multi-temporal InSAR algorithms are available- Persistent Scatterer (PS) and Small Baseline (SB) methods. This paper discusses the Stanford Method for Persistent Scatterer (StaMPS) based PS-InSAR and the Small Baselines Subset (SBAS) techniques to estimate the surface deformation in Tehri dam reservoir region in Uttarkhand, India. Both PS-InSAR and SBAS approaches used sixteen ENVISAT ASAR C-Band images for generating single master and multiple master interferograms stack respectively and their StaMPS processing resulted in time series 1D-Line of Sight (LOS) mean velocity maps which are indicative of deformation in terms of movement towards and away from the satellites. From 1D LOS velocity maps, localization of landslide is evident along the reservoir rim area which was also investigated in the previous studies. Both PS-InSAR and SBAS effectively extract measurement pixels in the study region, and the general results provided by both approaches show a similar deformation pattern along the Tehri reservoir region. Further, we conclude that StaMPS based PSInSAR method performs better in terms of extracting more number of measurement pixels and in the estimation of mean Line of Sight (LOS) velocity as compared to SBAS method. It is also proposed to take up a few major landslides area in Uttarakhand for slope stability assessment.
\end{abstract}

\section{INTRODUCTION}

Synthetic aperture radar (SAR) is an innovative technology of radar community. It is an active microwave remote sensing mechanism which is capable of imaging the earth in all weather conditions and making it valuable for several hazards monitoring application by measuring surface deformation. Initially, Interferometric SAR (InSAR) was adopted in several deformation monitoring applications by using two or more SAR images at different acquisition time to generate interferograms which after unwrapping produce Digital Elevation Model (DEM), and thereby determine the change in the position of resolution grids in the satellite line of sight (LOS). However, the reliability InSAR is always a concern because of temporal decorrelation and spatial decorrelation.

In order to overcome the aforementioned limitations of InSAR, time-series interferometric synthetic aperture radar (InSAR) methods are developed in the past to retrieve the deformation signal from pixels with different scattering characteristics. Presently, two classes of multi-temporal InSAR algorithms are available- Persistent Scatterer (PS) and Small Baseline (SB) methods. Ferretti et al. (2000) and Hooper et al. (2004) are credited with the most for the development of Permanent scatterer InSAR (PS-InSAR). PS-InSAR identifies measurement pixels known as permanent scatterer (PS) with stable amplitude and phase history over a long interval of time. Examples of such PS candidates are manmade objects such as buildings, roofs, etc. Although PS-InSAR is proved to be more accurate and consistent, it suffers from some limitations such as low PS density in non-urban areas and a relatively large temporal baseline (Greif and Vlcko, 2013).

In order to overcome these limitations, Hooper et al. (2007) developed Stanford Method of Persistent Scatterer (StaMPS) approach which uses spatial correlation of interferogram phase in defined neighbourhood to identify phase stable pixels over a period of time even with low amplitude stability which makes the approach capable of detecting PS pixels in non-urban areas. Apart from the PS, a few natural targets such as desert and noncultivated areas, often referred as DS with moderate coherence in few interferometric pairs, can also be explored to retrieve the timeseries deformation. These targets with moderate phase stability over complete observation period possess high spatial density compared to PS in non-urban areas. It has been observed that time-series of geophysical parameters can also be extracted from DS by reducing the decorrelation effect. This is achieved by forming interferograms between image pairs with small temporal baselines and a small difference in look angles. This is the working principle of the Small Baseline Subset (SBAS) approach which has been extensively exploited for measuring the surface deformation.

In the past, several researches have investigated the efficacy of PS-InSAR and SBAS in measuring surface deformation (Crosetto et al., 2005; Berardino et al., 2008; Hooper et al., 2007; Hooper et al., 2008; Agram, 2011; Tiwari et al., 2016). In this research work, we have implemented StaMPS based PS-InSAR and SBAS processing for analysing the slope instability in Tehri reservoir regions in Uttarakhand, India.

\footnotetext{
*Corresponding Author (ramjid@mnnit.ac.in)
} 


\section{STUDY AREA AND SATELLITE DATASET}

Landslide is the one of the most threatening geo-hazard of the Himalaya causing colossal damages to the infrastructure and livelihood of common people. Therefore, the Himalyan town of Tehri of Uttarakhand (India) is investigated in this study. Tehri town is inhabited near Tehri reservoir at the union of Bhagirathi and Bhilangana rivers. It is located at an altitude of $1,750 \mathrm{~m}$ $(5,740 \mathrm{ft})$. Tehri Dam is among the largest dams in India. In the past, several causalities haves been reported because of the landslides in this area. In December 2010, debris from the landslip has blocked a division tunnel forcing closure of electricity generation. This has caused a loss of more than 15 Million USD. Hence, the study is quite significant for this area.

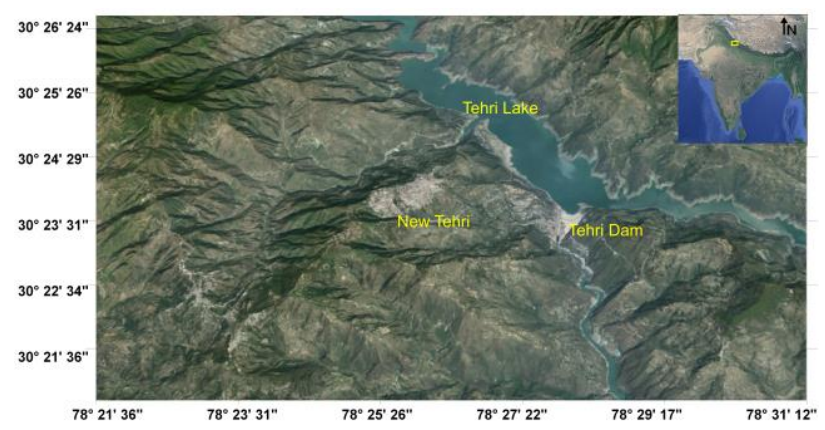

Fig. 1: Study area location (X-axis represent latitude and $\mathrm{Y}$-axis represent longitude)

\subsection{Satellite Dataset}

To monitor the critical slopes around Tehri reservoir, 16 Envisat ASAR C-Band SLC images of track 291 (frame: 83) acquired between January 2009 to July 2010 are used. ASAR image of $9^{\text {th }}$ October 2009 is chosen as the master image based on minimizing the temporal, Doppler and perpendicular baseline. The dataset is presented in Table 1.1 along with the critical perpendicular baseline length with respect to master image, acquisition date, orbit number and Doppler centroid frequency. Apart from the SLC images, a $90 \mathrm{~m}$ resolution SRTM Digital Elevation Model (DEM), in Figure 1, is used to remove the topographic phase from the differentially corrected interferogram. The DORIS precise orbits for the year 2009 and 2010 are used to remove orbital bias.

\begin{tabular}{|l|c|l|r|r|}
\hline $\begin{array}{l}\text { S. } \\
\text { No }\end{array}$ & $\begin{array}{l}\text { Acquisition } \\
\text { date }\end{array}$ & $\begin{array}{l}\text { Orbit } \\
\text { No. }\end{array}$ & $\mathbf{B}_{\perp}(\mathbf{m})$ & $\begin{array}{l}\text { Doppler } \\
\text { Centroid (Hz) }\end{array}$ \\
\hline 1. & 12Jan 2009 & 36417 & 301.6 & 172.014 \\
\hline 2. & 16Feb 2009 & 36417 & 305.1 & 348.914 \\
\hline 3. & 23Mar2009 & 36918 & 651.1 & 169.777 \\
\hline 4. & 27Apr 2009 & 37419 & 251.1 & 165.833 \\
\hline 5. & 01Jun 2009 & 37920 & 390.4 & 171.174 \\
\hline 6. & 06Jul 2009 & 38421 & 207.8 & 173.297 \\
\hline 7. & 10Aug2009 & 38922 & 173.2 & 173.608 \\
\hline 8. & 14Sep2009 & 39423 & 598.7 & 176.089 \\
\hline 9. & 19Oct2009 & $\mathbf{3 9 9 2 4}$ & $\mathbf{0}$ & 169.287 \\
\hline 10 & 23Nov2009 & 40924 & 50.33 & 172.812 \\
\hline 11 & 01Feb2010 & 41425 & 567.8 & 174.730 \\
\hline 12 & 08Mar2010 & 41427 & 112.3 & 177.697 \\
\hline 13 & 12Apr2010 & 42429 & 390 & 184.121 \\
\hline 14 & 17May2010 & 42930 & 426.9 & 166.537 \\
\hline 15 & 21Jun 2010 & 43431 & 273.4 & 190.413 \\
\hline 16 & 26Jul 2010 & 43932 & -27.9 & 186.428 \\
\hline
\end{tabular}

Table 1. ENVISAT data set of Tehri region

\section{STAMPS BASED PS-INSAR AND SBAS APPROACH}

The PS-InSAR method was initially developed by Ferretti et al. (2000). Later, Hanssen (2003), Lyons and Sandwell (2005) and Crossetto et al. (2005) proposed several modification and succeeded in finding PSs in urban areas which required a minimum of 15-20 interferograms for estimating the time series displacement of each detected PS pixel. The StaMPS method, developed by Hooper et al. (2007) came as an improvement to the above mentioned methods in the sense that the method is capable of finding PS pixels in urban as well as nonurban areas and also less number of interferograms is sufficient to map the surface displacement.

The interferogram generation in StaMPS is done using a single master image and is based on the maximization of the correlation amongst the set of images used for processing. The interferograms are then generated by using this master image which involves coregistration, resampling and complex multiplication of the master with all the slave images. Once interferograms are generated, the phase stability test is applied to obtain a set of phase stable pixels. An initial amplitude based filtering is performed using the amplitude dispersion index ' $D_{A}$ ' value which is the ratio of the standard deviation $\left(\sigma_{A}\right)$ and the mean $\left(\mu_{A}\right)$ of the amplitude values to filter the pixels which are not PS candidates. The topographical correction is done using an external DEM to convert the interferograms into differential interferograms, which are suitable for PS processing. The differential interferograms are input to the next step.

The pixels are initially selected on the basis of amplitude stability, in which those pixels which have a value of the amplitude dispersion index $D_{A}$ (ratio of the standard deviation and the mean of amplitude values) within the threshold are selected as initial PS candidates.

The candidates are tested for phase stability using a measure $\gamma_{x}$ stated in the following equation Hooper et al. (2007):

$$
\gamma_{x}=\frac{1}{N}\left|\sum_{i=1}^{N} \exp \left\{\sqrt{-1\left(\varphi_{x, i}-\tilde{\varphi}_{x, i}-\Delta \hat{\phi}_{\theta_{\theta, x, i}}^{u}\right)}\right\}\right|
$$

$N$ represents the number of interferograms, $\varphi_{x, i}$ represents the wrapped phase value of the $x^{\text {th }}$ pixel in the $i^{\text {th }}$ interferogram, $\tilde{\varphi}_{x, i}$ is the mean value of $\varphi_{x, i}$ and $\Delta \hat{\phi}_{\theta_{\theta, x, i}^{u}}$ represents the change in the spatially uncorrelated part of the look angle error for the $x^{\text {th }}$ pixel in the $i^{\text {th }}$ interferogram.

The pixels which satisfy the convergence of $\gamma_{x}$ to the threshold value are picked as PS pixels. The selected PS pixels contain a wrapped phase value, which is to be unwrapped, i.e. they must be added by an estimated number of phase cycles of $2 \pi$ to retrieve the original phase value, a process known as phase unwrapping. Other nuisance terms, such as the master and atmospheric error terms, spatially uncorrelated look angle error, satellite orbit errors are also estimated and removed from the unwrapped phase ' $\phi_{x, i}$ ' of the detected PS pixels. The displacement can then be estimated using the phase values of the individual PS pixels. A 1D Line of Sight (LOS) velocity map is generated as an output of the StaMPS method. 
However, StaMPS based Small Baseline Subset (SBAS) approach uses multiple master interferograms prepared based on reduction of spatial and temporal baseline. The method uses the amplitude difference dispersion index $\left(D_{\Delta \mathrm{A}}\right)$ given in equation as the criterion for selecting initial candidates.

$$
D_{\Delta A}=\frac{\sigma_{\Delta A}}{\mu_{A}}
$$

Where, $\sigma_{\Delta A}$ denotes the standard deviation of the amplitude differences and $\mu_{\mathrm{A}}$ is mean of the amplitude values of the respective pixel in the set of SLC images. $D_{\triangle A}$ is an indicator of phase stability when spectral filtering is applied in range, since it includes only the retained parts of the interferogram spectra (Hooper, 2008). The key difference in SBAS processing chain in comparison to PS-InSAR is the use of filtering to minimize phase decorrelation.

Similar to PS-InSAR, the phase stability estimation in SBAS approach makes use of the common low pass and adaptive filters to detect the atmospheric, topographical and orbital errors present in the phase of the candidate pixels. Once the phase stability step is completed, SB pixels are selected based on both the $\gamma$ and $D_{\Delta \mathrm{A}}$ value following equation (1). The Spatially Correlated Look Angle (SCLA) error and the Spatially Uncorrelated Look Angle (SULA) or (DEM) error are estimated in a similar manner as in case of the PS method. After phase unwrapping, the phase of the SB interferograms is inverted using the least squares method in order to estimate the phase due to deformation. The SCLA error is removed after phase unwrapping. Once all the errors are estimated and removed, the remaining phase value is considered as the phase due to deformation. The output of StaMPS based SBAS method, similar to PS-InSAR method, results in the form of a 1D LOS velocity map showing time series displacement of the extracted pixels. Detailed explanation of this approach can be found in Hooper et al. (2004, 2007), Hooper (2008).

\section{RESULTS AND DISCUSSION}

In this section, results of StaMPS based PS-InSAR processing of the 16 Envisat ASAR SLC images of Tehri and its adjoining area is presented. The parameters used for StaMPS based PSInSAR processing are shown in Table 2. PS-InSAR processing using 15 geocoded single master interferograms resulted in more than $12,50,000$ PS candidates based on the $D_{A}$ value in each patch with the area being divided into 6 patches which finally resulted in 19,549 PS pixels. The PS velocity plot (shown in Figure 3) shows displacement from -17.9 to 23.4 $\mathrm{mm} /$ year where cold and warm colours show displacement away and towards the satellite LOS respectively.

The StaMPS based SBAS approach produces multiple master small baseline interferograms and selection of a measurement pixel depends on the extent of decorrelation present in the study area. The StaMPS based SBAS approach resulted in a dense network of well-connected 107 interferograms. Finally, measurement pixels are extracted as measurement pixels by this method. Here, we observe that the SBAS method extracted less number of pixels (2545) than the PS-InSAR method owing to the fact that both are optimized for different kind of scattering models.
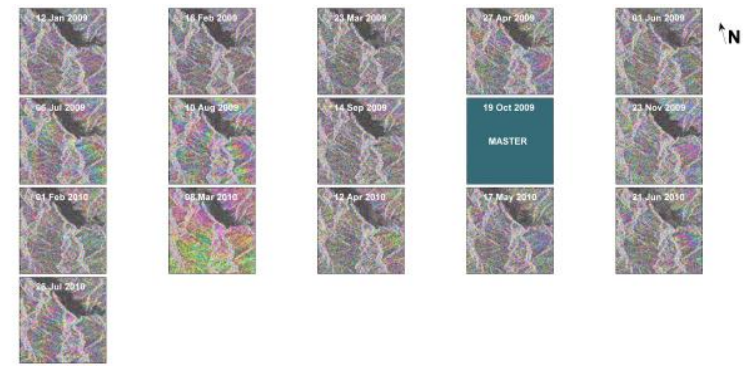

Fig. 2 Interferograms for the Tehri region. The image acquired on 19th October 2009 is chosen as the master image.

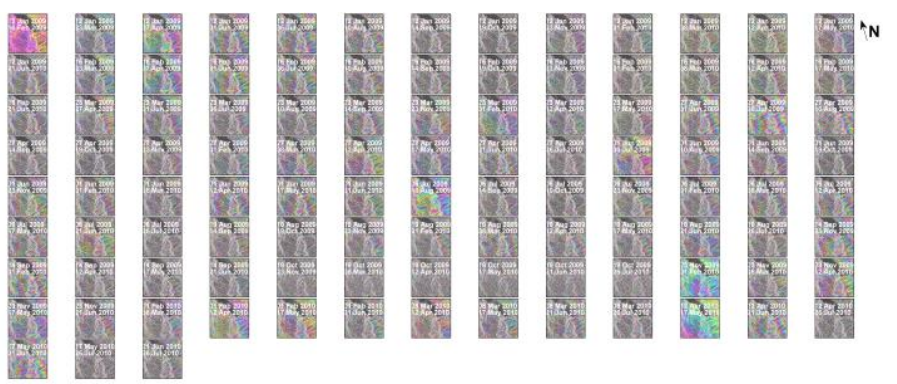

Fig. 3. Interferograms generated for SBAS processing.

\begin{tabular}{|l|l|c|}
\hline $\begin{array}{l}\text { S. } \\
\text { No }\end{array}$ & Parameters & $\begin{array}{l}\text { Value of } \\
\text { parameter }\end{array}$ \\
\hline $\mathbf{1}$ & No of images (N) & 16 \\
\hline $\mathbf{2}$ & Pixel grid size & 50 \\
\hline $\mathbf{3}$ & $\begin{array}{l}\text { Amplitude dispersion } \\
\text { threshold for further } \\
\text { processing in PS-InSAR chain }\end{array}$ & 0.45 \\
\hline $\mathbf{4}$ & Rate of convergence & 0.005 \\
\hline $\mathbf{5}$ & Number of patches in azimuth & 3 \\
\hline $\mathbf{6}$ & Number of patches in range & 2 \\
\hline $\mathbf{7}$ & $\begin{array}{l}\text { Overlapping pixel between } \\
\text { patches in azimuth }\end{array}$ & 200 \\
\hline
\end{tabular}

Table 2. Parameters for PS-InSAR processing of Tehri

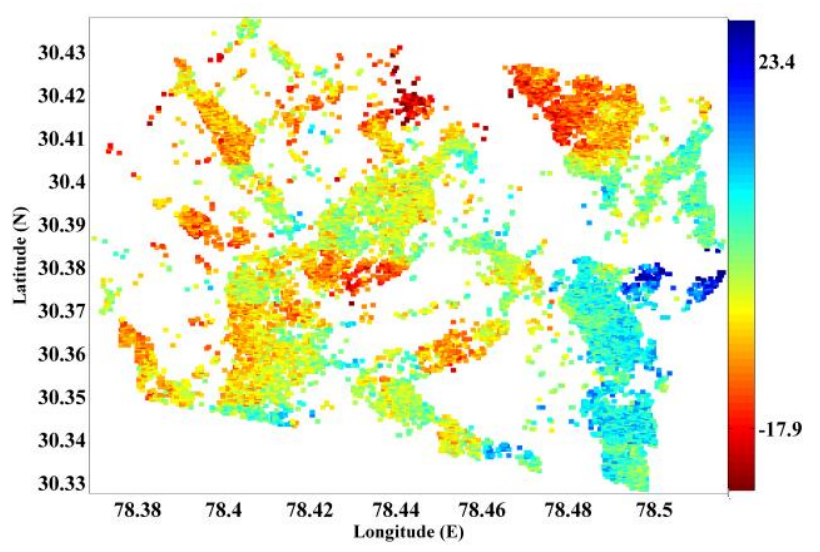

Fig. 4. Time series velocity plot of Tehri region showing PS pixel (colour bar showing velocity in $\mathrm{mm} / \mathrm{yr}$ ) 


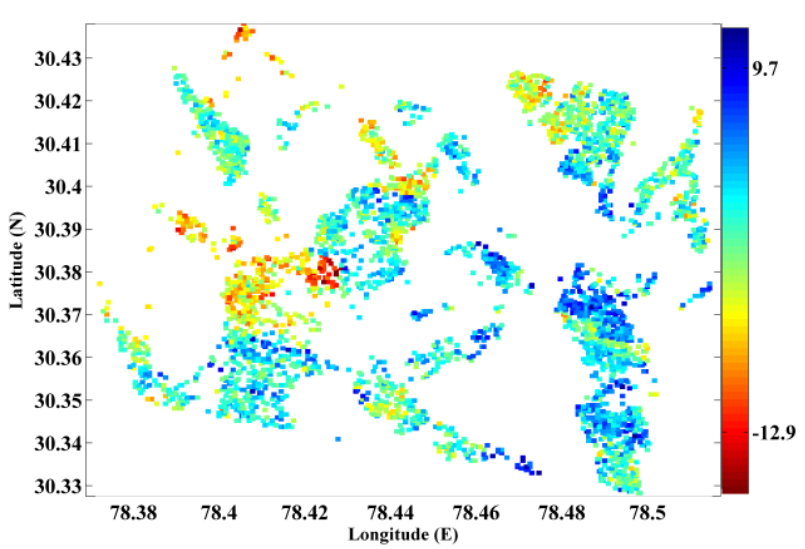

Fig. 5. Time series velocity plot of Tehri region showing SB pixels (colour bar showing velocity in $\mathrm{mm} / \mathrm{yr}$ )

This implies that there are more ground resolution elements in the study area which exhibit the characteristics of PS than slowly decorrelating scatterrers. The 1D LOS velocity map (Fig. 5) obtained from the StaMPS based SBAS method shows displacement rate in the range from -12.9 to $9.7 \mathrm{~mm} /$ year in cold and warm colors (color depiction similar to PS velocity map). The variation in mean velocity range may be due to the fact that the phase of the selected pixels obtained from both the methods is different. The inability of the SBAS method to detect sufficient number of measurement pixels makes the approach less effective in comparison to PS-InSAR. In the time series displacement plot (Fig. 5), there are regions which clearly show warm colors. Localization of landslide is evident from these patches with warm colors. However, the magnitudes of velocity of the extracted measurement pixels are different. This is due to the fact that the SBAS method uses filtering on the interferograms while PS-InSAR does not. This result in different deformation magnitudes for the same pixel if picked up by both the methods because the pixel phase values retrieved from both the methods may differ. These regions are adjoining the Tehri reservoir necessitating implementation of preventive measures to avoid any calamity in future.

\section{REFERENCES}

Agram, P., 2011. Comparison of Persistent Scatterers and Small Baseline Time-Series InSAR Results: A Case Study of the San Francisco Bay Area, IEEE Transactions on Geoscience and Remote Sensing, 8 (4): 592-596.

Berardino, P., Fornaro, G., Lanari, R., and Sansosti, G., 2002. A new algorithm for surface deformation monitoring using Small Baseline differential SAR Interferograms, IEEE Transactions on Geoscience and Remote Sensing, 40 (11): 2375-2383.

Crosetto, M., Crippa, B., and Biescas, E., 2005. Early detection and in-depth analysis of deformation phenomena by radar interferometry. Engineering Geology, 79(1-2): 81-91.

Ferretti, A., Prati, C., and Rocca F., 2000. Nonlinear subsidence rate estimation using Permanent Scatterers in differential SAR, IEEE Transactions on Geoscience and Remote Sensing, 38 (5): 2202 -2212.

Hanssen, R.F., 2003. Subsidence monitoring using contiguous and PS-InSAR: Quality assessment based on precision and reliability, Proceedings, 11th FIG Symposium on Deformation Measurements, Santorini, Greece.
Hooper, A., Zebker, H., Segall, P., and Kampes, B., 2004. A new method for measuring deformation on volcanoes and other natural terrains using InSAR persistent Scatterers, Geophysical Research Letters, 31 (23).

Hooper, A., Segall, P., and Zebker, H., 2007. Persistent Scatterer InSAR for Crustal Deformation Analysis, with Application to Volcan Alcedo, Galapagos, Journal of Geophysical Research 34.

Hooper, A., 2008. A multi-temporal InSAR method incorporating both persistent scatterer and small baseline approaches, Geophysical Research Letters, 35.

Greif, V., and Vlcko, J., 2013. "Application of the PS-InSAR Technique for the Post-Failure Landslide Deformation Monitoring at Lubietova Site in Central Slovakia" Landslide Science and Practice 2: 299-301.

Lyons, S., and Sandwell, D., 2003. Fault creep along the southern San Andreas from interferometric synthetic aperture radar, permanent scatterers, and stacking, Journal of Geophysical Research, 108(B1): 2047-2070.

Tiwari, A., Dwivedi, R., Dikshit, O., and Singh, A.K., 2016. A study on measuring surface deformation of L'Aquila region using StaMPS technique, International Journal of Remote Sensing, 37(4): $819-830$ 